\title{
Solving American Option Pricing Models by the Front Fixing Method: Numerical Analysis and Computing
}

\author{
R. Company, V. N. Egorova, and L. Jódar \\ Instituto de Matemática Multidisciplinar, Universitat Politécnica de València, Camino, de Vera s/n, 46022 Valencia, Spain \\ Correspondence should be addressed to V. N. Egorova; egorova.vn@gmail.com
}

Received 12 December 2013; Revised 3 April 2014; Accepted 6 April 2014; Published 28 April 2014

Academic Editor: Carlos Vazquez

Copyright ( 2014 R. Company et al. This is an open access article distributed under the Creative Commons Attribution License, which permits unrestricted use, distribution, and reproduction in any medium, provided the original work is properly cited.

\begin{abstract}
This paper presents an explicit finite-difference method for nonlinear partial differential equation appearing as a transformed BlackScholes equation for American put option under logarithmic front fixing transformation. Numerical analysis of the method is provided. The method preserves positivity and monotonicity of the numerical solution. Consistency and stability properties of the scheme are studied. Explicit calculations avoid iterative algorithms for solving nonlinear systems. Theoretical results are confirmed by numerical experiments. Comparison with other approaches shows that the proposed method is accurate and competitive.
\end{abstract}

\section{Introduction}

American options are contracts allowing the holder the right to sell (buy) an asset at a certain price at any time until a prespecified future date. The pricing of American options plays an important role both in theory and in real derivative markets.

The American option pricing problem can be posed either as a linear complementarity problem (LCP) or a free boundary value problem. These two different formulations have led to different methods for solving American options.

The most algebraic approach of LCPs for American option pricing can be found in $[1,2]$ and the references therein.

In this paper we follow the free boundary value approach. McKean [3] and van Moerbeke [4] show that the valuation of American options constitutes a free boundary problem looking for a boundary changing in time to maturity, known as the optimal exercise boundary. The problem of finding optimal exercise boundary can be treated analytically or numerically. With respect to the analytical approach, Geske and Johnson [5] obtained a valuation formula for American puts expressed in terms of a series of compound-option functions. See also Barone-Adesi and Whaley [6], MacMillan [7], and Ju [8].

Numerical methods were initiated by Brennan and Schwartz [9] and the convergence of their finite difference method was proved by Jaillet et al. [10]. Other relevant works using finite difference methods are Hull and White [11], Duffy [12], Wilmott et al. [1], Forsyth and Vetzal [13], Tavella and Randall [14], Tangman et al. [15], Zhu and Chen [16], and Kim et al. [17].

Free boundary value problems are challenging because one has to find the solution of a partial differential equation that satisfies auxiliary initial conditions and boundary conditions on a fixed boundary as well as on an unknown free boundary. This complexity is reduced by transforming the problem into a new nonlinear partial differential equation where the free boundary appears as a new variable of the PDE problem.

This technique which originated in physics problems is the so called front fixing method based on Landau transform [18] to fix the optimal exercise boundary on a vertical axis. The front fixing method has been applied successfully to a wide range of problems arising in physics (see [19]) and references therein. In 1997, Wu and Kwok [20] proposed the front fixing technique for solving free boundary problem to the field of option pricing. Other relevant papers related to the fixed domain transformation are Nielsen et al. [21], Ševčovič [22], and Zhang and Zhu [23]. All these papers use finite difference schemes after the corresponding fixed domain transformations. Nielsen et al. [21] use both explicit and implicit schemes while Ševčovič [22] uses difference schemes of operator splitting type. 
Wu and Kwok [20] transform the original problem into one more manageable equation because the coefficients of the transformed equation do not depend on the spatial variable. In [20], one uses a tree-level scheme and performs numerical tests versus the binomial method. The drawback of the initialization and iteration of the three-level method is overcome by Zhang and Zhu [23] using a predictor-corrector approach.

The front fixing method combined with the use of an explicit finite difference scheme avoids the drawbacks of alternative algebraic approaches since it avoids the use of iterative methods and underlying difficulties such as how to initiate the algorithm, when to stop it, and which is the error after the stopping.

As the best model can be wasted with a disregarded numerical analysis, in this paper, we focus on the numerical issues of explicit finite difference scheme for American put option problem after using the fixed domain transformation used in [20]. Consistency of the numerical scheme with the PDE problem is stated. Conditions on the size of discretization steps in order to guarantee stability and positivity of the numerical solution are given. Furthermore, the numerical solution preserves properties of the theoretical solution presented in [24], such as the positivity and the monotonicity of the optimal exercise boundary.

This paper is organized as follows. In Section 2, dimensionless fixed domain transformation of the American put option problem is introduced as well as the discretization of the continuous problem. Section 3 is addressed to guarantee the positivity of the numerical solution as well as the nonincreasing time behaviour of the optimal exercise boundary. In Section 4, stability and consistency are treated. Finally, in Section 5, numerical experiments and comparison with other methods are illustrated.

Throughout the paper we will denote for a given $x=$ $\left[x_{1}, x_{2}, \ldots, x_{N}\right]^{T} \in \mathbb{R}^{N}$ its supremum norm as $\|x\|_{\infty}=$ $\max \left\{\left|x_{i}\right|: 1 \leq i \leq N\right\}$.

\section{Fixed Domain Transformation and Discretization}

It is well-known that the American put option price model is given by [1] the moving free boundary PDE as follows:

$$
\frac{\partial P}{\partial \tau}=\frac{1}{2} \sigma^{2} S^{2} \frac{\partial^{2} P}{\partial S^{2}}+r S \frac{\partial P}{\partial S}-r P, \quad S>B(\tau), 0<\tau \leq T,
$$

together with the initial and boundary conditions:

$$
\begin{gathered}
P(S, 0)=\max (E-S, 0), \quad S \geq 0, \\
\frac{\partial P}{\partial S}(B(\tau), \tau)=-1, \\
P(B(\tau), \tau)=E-B(\tau), \\
\lim _{S \rightarrow \infty} P(S, \tau)=0,
\end{gathered}
$$

$$
\begin{gathered}
B(0)=E, \\
P(S, \tau)=E-S, \quad 0 \leq S<B(\tau),
\end{gathered}
$$

where $\tau=T-t$ denotes the time to maturity $T, S$ is the asset's price, $P(S, \tau)$ is the option price, $B(\tau)$ is the unknown early exercise boundary, $\sigma$ is a volatility of the asset, $r$ is the riskfree interest rate, and $E$ is the strike price.

Note that if asset price $S \leq B(\tau)$, the optimal strategy is to exercise the option, while if $S>B(\tau)$, the optimal strategy is to hold the option.

Let us consider the dimensionless change of the following variables [20]:

$$
p(x, \tau)=\frac{P(S, \tau)}{E}, \quad S_{f}(\tau)=\frac{B(\tau)}{E}, \quad x=\ln \frac{S}{S_{f}(\tau)},
$$

that transforms the problem (1)-(2) into the normalized form as follows:

$$
\begin{array}{r}
\frac{\partial p}{\partial \tau}=\frac{1}{2} \sigma^{2} \frac{\partial^{2} p}{\partial x^{2}}+\left(r-\frac{\sigma^{2}}{2}\right) \frac{\partial p}{\partial x}-r p+\frac{S_{f}^{\prime}}{S_{f}} \frac{\partial p}{\partial x} \\
x>0, \quad 0<\tau \leq T,
\end{array}
$$

where $S_{f}^{\prime}$ denotes the derivative of $S_{f}$ with respect to $\tau$. The new boundary and initial conditions are

$$
\begin{gathered}
p(x, 0)=0, \quad x \geq 0, \\
\frac{\partial p}{\partial x}(0, \tau)=-S_{f}(\tau), \\
p(0, \tau)=1-S_{f}(\tau), \\
\lim _{x \rightarrow \infty} p(x, \tau)=0, \\
S_{f}(0)=1 .
\end{gathered}
$$

The Equation (4) is a nonlinear differential equation on the domain $(0, \infty) \times(0, T]$. In order to solve numerically problem (4)-(9), one has to consider a bounded numerical domain. Let us introduce $x_{\max }$ large enough to translate the boundary condition (8); that is, $p\left(x_{\max }, \tau\right)=0$. Then the problem (4)-(9) can be studied on the fixed domain $\left[0, x_{\max }\right] \times(0, T]$. The value $x_{\max }$ is chosen following the criterion pointed out in [25].

Let us introduce the computational grid of $M+1$ space points and $N$ time levels with respective stepsizes $h$ and $k$ as follows:

$$
\begin{gathered}
h=\frac{x_{\max }}{M+1}, \quad k=\frac{T}{N}, \\
x_{j}=h j, \quad j=0, \ldots, M+1, \\
\tau^{n}=k n, \quad n=0, \ldots, N .
\end{gathered}
$$


The approximate value of $p(x, \tau)$ at the point $x_{j}$ and time $\tau^{n}$ is denoted by $p_{j}^{n} \approx p\left(x_{j}, \tau^{n}\right)$. Then a forward-time centralspace explicit scheme is constructed for internal spacial nodes as follows:

$$
\begin{aligned}
& \frac{p_{j}^{n+1}-p_{j}^{n}}{k}= \frac{1}{2} \sigma^{2} \frac{p_{j-1}^{n}-2 p_{j}^{n}+p_{j+1}^{n}}{h^{2}}+\left(r-\frac{\sigma^{2}}{2}\right) \frac{p_{j+1}^{n}-p_{j-1}^{n}}{2 h} \\
&-r p_{j}^{n}+\frac{S_{f}^{n+1}-S_{f}^{n}}{k S_{f}^{n}} \frac{p_{j+1}^{n}-p_{j-1}^{n}}{2 h}, \\
& 1 \leq j \leq M, \quad 0 \leq n \leq N-1 .
\end{aligned}
$$

By denoting $\mu=k / h^{2}$, the scheme (11) can be rewritten in the following form:

$$
p_{j}^{n+1}=a p_{j-1}^{n}+b p_{j}^{n}+c p_{j+1}^{n}+\frac{S_{f}^{n+1}-S_{f}^{n}}{2 h S_{f}^{n}}\left(p_{j+1}^{n}-p_{j-1}\right),
$$

where

$$
\begin{gathered}
a=\frac{\mu}{2}\left(\sigma^{2}-\left(r-\frac{\sigma^{2}}{2}\right) h\right) ; \quad b=1-\sigma^{2} \mu-r k ; \\
c=\frac{\mu}{2}\left(\sigma^{2}+\left(r-\frac{\sigma^{2}}{2}\right) h\right) .
\end{gathered}
$$

From the boundary conditions (6) and (7), we can obtain

$$
\frac{p_{1}^{n}-p_{-1}^{n}}{2 h}=-S_{f}^{n} ; \quad p_{0}^{n}=1-S_{f}^{n},
$$

where $x_{-1}=-h$ is an auxiliary point out of the domain. By considering (4) at the point $x_{0}=0, \tau>0$ (see [26], p. 341), and replacing of the boundary conditions (6) and (7) into (4) at $\left(0^{+}, \tau\right)$, one gets the new boundary condition as follows:

$$
\frac{1}{2} \sigma^{2} \frac{\partial^{2} p}{\partial x^{2}}\left(0^{+}, \tau\right)+\frac{\sigma^{2}}{2} S_{f}(\tau)-r=0
$$

(see $[20,23,26])$, and its central difference discretization is

$$
\frac{\sigma^{2}}{2} \frac{p_{1}^{n}-2 p_{0}^{n}+p_{-1}^{n}}{h^{2}}+\frac{\sigma^{2}}{2} S_{f}^{n}-r=0
$$

From (14) and (16), the value of $p_{-1}^{n}$ can be eliminated obtaining the relationship

$$
p_{1}^{n}=\alpha-\beta S_{f}^{n}, \quad n \geq 1,
$$

between the free boundary approximation $S_{f}^{n}$ and $p_{1}^{n}$, where

$$
\alpha=1+\frac{r h^{2}}{\sigma^{2}}, \quad \beta=1+h+\frac{1}{2} h^{2} .
$$

By using the scheme (12) for $j=1$ and evaluating (17) at the $(n+1)^{\text {st }}$ time level, the free boundary $S_{f}^{n+1}$ can be expressed as

$$
S_{f}^{n+1}=d^{n} S_{f}^{n}, \quad 0 \leq n \leq N-1,
$$

where

$$
d^{n}=\frac{\alpha-\left(a p_{0}^{n}+b p_{1}^{n}+c p_{2}^{n}-\left(p_{2}^{n}-p_{0}^{n}\right) / 2 h\right)}{\left(p_{2}^{n}-p_{0}^{n}\right) / 2 h+\beta S_{f}^{n}} .
$$

After expression (19), the value $S_{f}^{n+1}$ can be replaced in (12), (17), and (14) to obtain values $p_{j}^{n+1}, 0 \leq j \leq M$. Then the numerical scheme for the problem (4)-(9) can be rewritten for any $n=0, \ldots, N-1$ in the following algorithmic form:

$$
\begin{gathered}
S_{f}^{n+1}=d^{n} S_{f}^{n}, \\
p_{0}^{n+1}=1-S_{f}^{n+1}, \\
p_{1}^{n+1}=\alpha-\beta S_{f}^{n+1}, \\
p_{j}^{n+1}=\widetilde{a}^{n} p_{j-1}^{n}+b p_{j}^{n}+\widetilde{c}^{n} p_{j+1}^{n}, \quad j=2, \ldots, M,
\end{gathered}
$$

where

$$
\begin{gathered}
\widetilde{a}^{n}=a-\frac{S_{f}^{n+1}-S_{f}^{n}}{2 h S_{f}^{n}}, \quad \widetilde{c}^{n}=c+\frac{S_{f}^{n+1}-S_{f}^{n}}{2 h S_{f}^{n}}, \\
p_{M+1}^{n+1}=0
\end{gathered}
$$

with the initial conditions

$$
S_{f}^{0}=1, \quad p_{j}^{0}=0, \quad 0 \leq j \leq M+1 .
$$

\section{Positivity and Monotonicity}

In this section, we will show the free boundary nonincreasing monotonicity as well as the positivity and nonincreasing spacial monotonicity of the numerical option price. Let us start this section by showing that constant coefficients $a$, $b$, and $c$ of scheme (12) for the transformed problem (4)(9) are positive under appropriate conditions on the stepsize discretization $h$ and $k$.

Lemma 1. Assuming that the stepsizes $h$ and $k$ satisfy the following conditions:

$$
\begin{aligned}
& C 1: h \leq \sigma^{2} /\left|r-\left(\sigma^{2} / 2\right)\right|, r \neq \sigma^{2} / 2, \\
& C 2: k \leq h^{2} /\left(\sigma^{2}+r h^{2}\right),
\end{aligned}
$$

then the coefficients of the scheme $a, b$, and $c$ are nonnegative. If $r=\sigma^{2} / 2$, then under the condition $C 2$, coefficients $a, b$, and $c$ are nonnegative.

Proof. From (13) for nonnegativity of $a$, it is necessary that

$$
\sigma^{2}-\left(r-\frac{\sigma^{2}}{2}\right) h \geq 0
$$

If $r \leq \sigma^{2} / 2$ from (13), note that $a \geq 0$ for any $h>0$. Otherwise, (27) is satisfied under the condition Cl.

From (13), $b$ is nonnegative under the condition C2.

If $r \geq \sigma^{2} / 2$, nonnegativity of $c$ is guaranteed by (13) for any $h>0$. Otherwise, $c$ is nonnegative under the condition $\mathrm{C} 1$ 
The following lemma prepares the study of positivity of the numerical solution $p_{j}^{n}$ as well as the monotonicity of the free boundary sequence $S_{f}^{n}$, that will be established in a further result.

Lemma 2. Let $\left\{p_{j}^{n}, S_{f}^{n}\right\}$ be the numerical solution of scheme (21)-(26) for a transformed American put option problem (4) and let $d^{n}$ be defined by (20). Then under hypothesis of the Lemma 1, for small enough $h$, one verifies the following.

(1) For each fixed n,

$$
0<d^{n} \leq 1 .
$$

(2) Values $p_{j}^{n+1} \geq 0$ for $j=0, \ldots, M ; n=0, \ldots, N-1$.

(3) $p_{j}^{n+1} \geq p_{j+1}^{n+1}$ for $j=0, \ldots, M-1 ; n=0, \ldots, N-1$.

Proof. We use the induction principle. Note that from (26), we have $p_{j}^{0} \geq 0$ and $p_{j}^{0} \geq p_{j+1}^{0}$. Consider the first time level $n=0$. From (18), (20), (26), and hypothesis C1 of Lemma 1, one gets

$$
0<d^{0}=\frac{\alpha}{\beta} \leq 1 .
$$

Note that from (21)-(23) and (29) one gets

$$
0<S_{f}^{1}=d^{0} \leq 1 ; \quad p_{0}^{1}=1-d^{0} \geq 0 ; \quad p_{1}^{1}=0,
$$

and from (24) and (26), every $p_{j}^{1}=0$ for $j=2, \ldots, M$.

For the sake of clarity, let us show firstly that $0<d^{1} \leq 1$. From (20), (18), and (30), it follows that

$$
\begin{aligned}
d^{1} & =1-a \frac{\beta-\alpha}{\alpha \beta-(\beta-\alpha) / 2 h} \\
& =1-a \frac{h+h^{2}\left(1 / 2-r / \sigma^{2}\right)}{1 / 2+h\left(3 / 4+r / 2 \sigma^{2}\right)+O\left(h^{2}\right)} .
\end{aligned}
$$

As $a$ is positive by Lemma 1 , for small enough values of $h$, one gets

$$
0<d^{1} \leq 1 .
$$

It is easy to show that $p_{2}^{2}=0$, and for $d^{2}$, analogously as (31) and (32), $0<d^{2} \leq 1$.

Let us assume the induction hypothesis that conclusions hold true for index $n-1$; that is,

$$
0<d^{n-1} \leq 1, \quad p_{j}^{n} \geq 0, \quad p_{j}^{n} \geq p_{j+1}^{n} .
$$

Now we are going to prove that conclusions hold true for index $n$. Let us consider value of $d^{n}$ for $n \geq 1$. By denoting

$$
\begin{gathered}
f^{n}=1+\frac{r h^{2}}{\sigma^{2}}-\left(a p_{0}^{n}+b p_{1}^{n}+c p_{2}^{n}-\frac{p_{2}^{n}-p_{0}^{n}}{2 h}\right), \\
g^{n}=\frac{p_{2}^{n}-p_{0}^{n}}{2 h}+\left(\frac{1}{2}\left(1+(1+h)^{2}\right)\right) S_{f}^{n},
\end{gathered}
$$

then from (20),

$$
d^{n}=\frac{f^{n}}{g^{n}}
$$

For $n>2$, using Taylor's expansion, the approximation of the involved derivatives, and boundary conditions (6), (7), and (15),

$$
p_{2}^{n}=1+\frac{4 r h^{2}}{\sigma^{2}}-\left(1+2 h+2 h^{2}\right) S_{f}^{n}+O\left(h^{3}\right) .
$$

From (37) and (34), numerator $f^{n}$ takes the form

$$
\begin{aligned}
f^{n}= & r h\left[k+\frac{r k h+2(1-r k)}{\sigma^{2}}\right] \\
& +\left(\frac{h^{2}}{2}(1-r k)-k h \frac{\sigma^{2}}{2}\right) S_{f}^{n}+O\left(h^{2}\right),
\end{aligned}
$$

and verifies $f^{n}>0$ since $k<h /\left(r h+\sigma^{2}\right)$ under condition C2 of lemma and for $h<1$.

From (37) and (20), denominator $g^{n}$ is positive for small enough values of $h$, since

$$
\begin{aligned}
g^{n} & =\frac{p_{2}^{n}-p_{0}^{n}}{2 h}+\left(1+h+\frac{h^{2}}{2}\right) S_{f}^{n} \\
& =\frac{2 r h}{\sigma^{2}}+\frac{h^{2}}{2} S_{f}^{n}+O\left(h^{2}\right)>0 .
\end{aligned}
$$

From (36) and previous comments, one gets $d^{n}>0$. In order to prove that $d^{n} \leq 1$, let us consider the difference $f^{n}$ $g^{n}$. By using (38) and (39) under hypothesis of Lemma 2, one can obtain

$$
f^{n}-g^{n}=k h\left(r \frac{\sigma^{2}-2 r+r h}{\sigma^{2}}-\frac{r h+\sigma^{2}}{2} S_{f}^{n}\right)+O\left(h^{2}\right) .
$$

Note that if $\sigma^{2}<2 r$, then (40) is nonpositive for small enough values of $h$. However, even if $\sigma \geq 2 r$, the Samuelson asymptotic limit [27] $S_{f}^{n} \geq 2 r /\left(2 r+\sigma^{2}\right)$ (see [20], page 87) guarantees the nonpositivity of (40). Therefore $d^{n} \leq 1$.

In order to prove the positivity of $\left\{p_{j}^{n+1}\right\}$, it will be useful to show the nonnegativity of coefficients $\widetilde{a}^{n}$ and $\widetilde{c}^{n}$ appearing in (21). Coefficient $\widetilde{a}^{n}$ is positive since

$$
\tilde{a}^{n}=a-\frac{S_{f}^{n+1}-S_{f}^{n}}{2 h S_{f}^{n}}=a-\frac{d^{n}-1}{2 h} \geq a \geq 0 .
$$

In order to show the positivity of the coefficient $\tilde{c}^{n}$, note that from (13) and (39), the sign of $\widetilde{c}^{n}$ is the same as the sign of $\left(2 h c g^{n}+f^{n}-g^{n}\right)$ and from (13), (38), (39), and $S_{f}^{n} \leq 1$, one gets the following for small enough values of $h$ :

$$
2 h c g^{n}+f^{n}-g^{n}>r k+\left(\sigma^{2} \mu+r k\right) \frac{r h^{2}}{\sigma^{2}}-\frac{k h^{2} \sigma^{2}}{4}>0 .
$$


Under hypotheses of induction (33) together with positivity of coefficients $\widetilde{a}^{n}$ and $\widetilde{c}^{n}$, the positivity of $\left\{p_{j}^{n+1}\right\}$ is proved. Moreover, $\left\{p_{j}^{n+1}\right\}$ is nonincreasing with respect to index $j$ from (24), since

$$
\begin{aligned}
p_{j}^{n+1}-p_{j+1}^{n+1}= & \widetilde{a}^{n}\left(p_{j-1}^{n}-p_{j}^{n}\right)+b\left(p_{j}^{n}-p_{j+1}^{n}\right) \\
& +\widetilde{c}^{n}\left(p_{j+1}^{n}-p_{j+2}^{n}\right) \geq 0 .
\end{aligned}
$$

Summarizing, the following result has been established.

Theorem 3. Under assumptions of Lemma 2, the numerical scheme (12) for solving the American option transformed problem guarantees the following properties of the numerical solution:

(i) nonincreasing monotonicity and positivity of values $S_{f}^{n}$, $n=0, \ldots, N$

(ii) positivity of the vectors $p^{n}, n=0, \ldots, N$;

(iii) nonincreasing monotonicity of the vectors $p^{n}=$ $\left(p_{0}^{n}, \ldots, p_{M}^{n}\right)$ with respect to space indexes for each fixed $n=0, \ldots, N$.

\section{Stability and Consistency}

In this section, we study the stability and consistency properties of the scheme (21)-(26). For the sake of clarity in the presentation knowing that several different concepts of the stability are used in the literature, we begin the section with the following definition.

Definition 4 . The numerical scheme (11) is said to be $\|\cdot\|_{\infty}$ stable in the domain $\left[0, x_{\infty}\right] \times[0, T]$, if for every partition with $k=\Delta \tau, h=\Delta x, N k=T$, and $(M+1) h=x_{\infty}$,

$$
\left\|P^{n}\right\|_{\infty} \leq C, \quad 0 \leq n \leq N,
$$

where $C$ is independent of $h, k$, and $n=0, \ldots, N$ (see [28]).

Theorem 5. Under assumptions of Lemma 2, the numerical scheme (21)-(26) for solving transformed problem (4)-(9) is $\|\cdot\|_{\infty}$-stable.

Proof of Theorem 5. Since for each fixed $n,\left\{p_{j}^{n}\right\}$ is a nonincreasing sequence with respect to $j$, then according to the boundary condition (22) and positivity of $S_{f}^{n}$ since (28), one gets

$$
\left\|P^{n}\right\|_{\infty}=p_{0}^{n}=1-S_{f}^{n}<1, \quad 0 \leq n \leq N .
$$

Thus, the scheme is $\|\cdot\|_{\infty}$-stable.

Classical consistency of a numerical scheme with respect to a partial differential equation means that the exact theoretical solution of the PDE approximates well the solution of the difference scheme as the discretization stepsizes tend to zero [29]. However, in our case, we have to harmonize the behaviour not only of the PDE (4) with the scheme
(24), but also the boundary conditions (6), (7), and (15) with the numerical scheme for the free boundaries (21) and (20). With respect to the first part of consistency, let us write the numerical scheme (24) in the following form:

$$
\begin{aligned}
F( & \left.p_{j}^{n}, S_{f}^{n}\right) \\
= & \frac{p_{j}^{n+1}-p_{j}^{n}}{k}-\frac{1}{2} \sigma^{2} \frac{p_{j-1}^{n}-2 p_{j}^{n}+p_{j+1}^{n}}{h^{2}} \\
& \quad-\left(r-\frac{\sigma^{2}}{2}\right) \frac{p_{j+1}^{n}-p_{j-1}^{n}}{2 h}+r p_{j}^{n}-\frac{S_{f}^{n+1}-S_{f}^{n}}{k S_{f}^{n}} \frac{p_{j+1}^{n}-p_{j-1}^{n}}{2 h}
\end{aligned}
$$$$
=0 \text {. }
$$

Let us denote by $\widetilde{p}_{j}^{n}=p\left(x_{j}, \tau^{n}\right)$ the exact theoretical solution value of the PDE at the mesh point $\left(x_{j}, \tau^{n}\right)$, and let $\widetilde{S}_{f}^{n}=S_{f}\left(\tau^{n}\right)$ be the exact solution of the free boundary at time $\tau^{n}$. The scheme (46) is said to be consistent with

$$
\begin{aligned}
& L\left(p, S_{f}\right) \\
& \quad=\frac{\partial p}{\partial \tau}-\frac{1}{2} \sigma^{2} \frac{\partial^{2} p}{\partial x^{2}}-\left(r-\frac{\sigma^{2}}{2}\right) \frac{\partial p}{\partial x}+r p-\frac{S_{f}^{\prime}}{S_{f}} \frac{\partial p}{\partial x}=0,
\end{aligned}
$$

if the local truncation error

$$
T_{j}^{n}\left(\widetilde{p}, \widetilde{S}_{f}\right)=F\left(\widetilde{p}_{j}^{n}, \widetilde{S}_{f}^{n}\right)-L\left(\widetilde{p}_{j}^{n}, \widetilde{S}_{f}^{n}\right)
$$

satisfies

$$
T_{j}^{n}\left(\tilde{p}, \widetilde{S}_{f}\right) \longrightarrow 0, \quad \text { as } h \longrightarrow 0, k \longrightarrow 0 .
$$

Assuming the existence of the continuous partial derivatives up to order two in time and up to order four in space, using Taylor's expansion about $\left(x_{j}, \tau^{n}\right)$, one gets

$$
\begin{aligned}
T_{j}^{n}\left(\tilde{p}, \widetilde{S}_{f}\right) \\
=k E_{j}^{n}(3)-\frac{\sigma^{2}}{2} h^{2} E_{j}^{n}(2)+\left(r-\frac{\sigma^{2}}{2}\right) h^{2} E_{j}^{n}(1) \\
\quad-k E_{j}^{n}(4) \frac{\partial p}{\partial x}\left(x_{j}, \tau^{n}\right)-h^{2} E_{j}^{n}(1) \frac{1}{\widetilde{S}_{f}^{n}} \frac{d S_{f}}{d \tau}\left(\tau^{n}\right) \\
\quad-k h^{2} E_{j}^{n}(4) E_{j}^{n}(1),
\end{aligned}
$$

where

$$
\begin{aligned}
E_{j}^{n}(1)=\frac{1}{6} \frac{\partial^{3} p}{\partial x^{3}}\left(x_{j}, \tau^{n}\right), & E_{j}^{n}(2)=\frac{1}{12} \frac{\partial^{4} p}{\partial x^{4}}\left(x_{j}, \tau^{n}\right), \\
E_{j}^{n}(3)=\frac{1}{2} \frac{\partial^{2} p}{\partial \tau^{2}}\left(x_{j}, \tau^{n}\right), & E_{j}^{n}(4)=\frac{k}{2 \widetilde{S}_{f}} \frac{d^{2} S_{f}}{d \tau^{2}}\left(\tau^{n}\right) .
\end{aligned}
$$


Equations (50) and (51) show the local truncation error of the numerical scheme (24) with respect to the PDE (4). Note that from (50) and (51),

$$
T_{j}^{n}\left(\widetilde{p}, \widetilde{S}_{f}\right)=O\left(h^{2}\right)+O(k) .
$$

In order to complete the consistency of the solution of the free boundary problem with the scheme (21)-(26), it is necessary to rewrite the boundary conditions (6), (7), and (15) in the following form:

$$
\begin{gathered}
L_{1}\left(p, S_{f}\right)=p(0, \tau)-1+S_{f}(\tau)=0, \\
L_{2}\left(p, S_{f}\right)=\frac{\partial p}{\partial x}(0, \tau)+S_{f}(\tau)=0, \\
L_{3}\left(p, S_{f}\right)=\frac{\sigma^{2}}{2} \frac{\partial^{2} p}{\partial x^{2}}(0, \tau)+\frac{\sigma^{2}}{2} S_{f}(\tau)-r=0 .
\end{gathered}
$$

Furthermore, we have finite difference approximation for the following boundary conditions:

$$
\begin{gathered}
F_{1}\left(p, S_{f}\right)=p_{0}^{n}-1+S_{f}^{n}=0, \\
F_{2}\left(p, S_{f}\right)=\frac{p_{1}^{n}-p_{-1}^{n}}{2 h}+S_{f}^{n}=0, \\
F_{3}\left(p, S_{f}\right)=\frac{\sigma^{2}}{2} \frac{p_{-1}^{n}-2 p_{0}^{n}+p_{1}^{n}}{h^{2}}+\frac{\sigma^{2}}{2} S_{f}^{n}-r=0 .
\end{gathered}
$$

It is not difficult to check using Taylor's expansion that the local truncation error satisfies

$$
\begin{gathered}
T_{1}\left(\tilde{p}, \widetilde{S}_{f}\right)=F_{1}\left(\tilde{p}, \widetilde{S}_{f}\right)-L_{1}\left(\tilde{p}, \widetilde{S}_{f}\right)=0, \\
T_{2}\left(\tilde{p}, \widetilde{S}_{f}\right)=F_{2}\left(\tilde{p}, \widetilde{S}_{f}\right)-L_{2}\left(\tilde{p}, \widetilde{S}_{f}\right)=O\left(h^{2}\right), \\
T_{3}\left(\tilde{p}, \widetilde{S}_{f}\right)=F_{3}\left(\tilde{p}, \widetilde{S}_{f}\right)-L_{3}\left(\tilde{p}, \widetilde{S}_{f}\right)=O\left(h^{2}\right) .
\end{gathered}
$$
as $h^{2}$.

The truncation error for the boundary condition behaves

Theorem 6. Assuming that the solution of the PDE problem (4)-(9) admits two times continuous partial derivative with respect to time and up to order four with respect to space, the numerical solution computed by the scheme (21)-(26) is consistent with (4) and boundary conditions of order two in space and order one in time.

\section{Numerical Experiments}

In this section we illustrate the previous theoretical results with numerical experiments showing the potential advantages of the proposed method such as preserving the qualitative properties of the theoretical solution [24] such as positivity and monotonicity. A comparison with other approaches is also presented in this section.

5.1. Confirmations of Theoretical Results. In this experiment we check that the stability condition can be broken showing

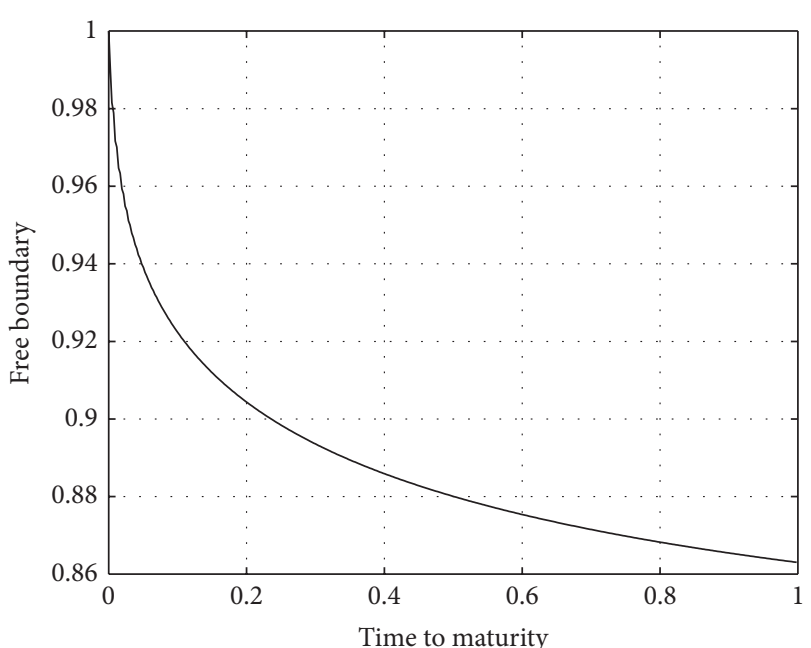

(a)

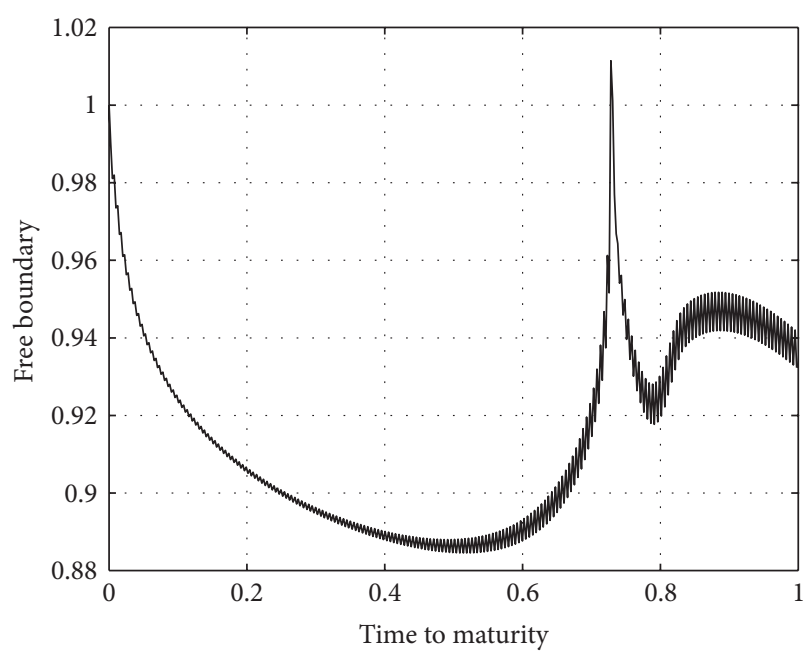

(b)

FIGURE 1: Dependence of stability on positivity of coefficient $b$. ( $\mu=$ $24, b=0.0398)$ for (a) and ( $\mu=25.2, b=-0.0083)$ for (b).

that in such case the results could become unreliable. Let us consider an American put option pricing problem as in [21] with the following parameters:

$$
r=0.1, \quad \sigma=0.2, \quad T=1, \quad x_{\max }=2 .
$$

We consider fixed space step $h=0.01$ that satisfies condition $\mathrm{C} 1$ of Lemma 1, and change time step (Figure 1). Numerical tests show that the condition C2 is critical for positivity of coefficient $b$ and, as a result, for the stability of the scheme. The monotonicity of the free boundary is numerically shown by numerical tests (Figure 1(a)). In Figure 1(b), one can see that when condition C2 is not satisfied, spurious oscillations appear so the monotonicity is broken.

It was theoretically proved in Section 4 that the scheme has order of approximation $O\left(h^{2}\right)+O(k)$. The results of the 
TABLE 1: Convergence in space for fixed time step $k=2 \cdot 10^{-3}$ for the problem with parameters (56).

\begin{tabular}{lcccc}
\hline Asset price & True value & 0.04 & 0.02 & 0.01 \\
\hline 90 & 11.6974 & 11.7283 & 11.7054 & 11.6991 \\
100 & 6.9320 & 6.9308 & 6.9309 & 6.9312 \\
110 & 4.1550 & 4.1694 & 4.1564 & 4.1531 \\
120 & 2.5102 & 2.5219 & 2.5151 & 2.5114 \\
& RMSE & $1.8037-2$ & $4.7857-3$ & $1.4623-3$ \\
& CPU-time (sec) & 0.052 & 0.075 & 0.105 \\
\hline
\end{tabular}

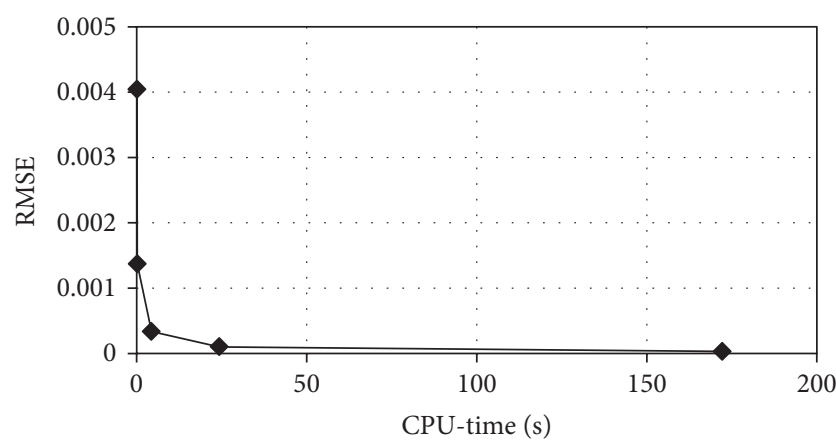

FIGURE 2: RMSE against the computational time.

numerical experiments for the problem with the following parameters:

$$
r=0.08, \quad \sigma=0.2, \quad T=3, \quad x_{\max }=2
$$

are presented in Table 1. For "True" values, we use the reference values offered in [30]. We consider the different space steps for fixed time step $k=0.002$ to guarantee the stability. The root-mean-square error (RMSE) is used to measure the accuracy of the scheme. The last row presents the CPU-time in seconds for each experiment. The plot of the RMSE against computational time is presented in Figure 2.

To check the order of approximation in space, we introduce the convergence rate

$$
\gamma\left(h_{1}, h_{2}\right)=\frac{\ln \mathrm{RMSE}_{h_{1}}-\ln \mathrm{RMSE}_{h_{2}}}{\ln h_{1}-\ln h_{2}} .
$$

From Table 1 and (58), one obtains $\gamma\left(4 \cdot 10^{-2}, 2 \cdot 10^{-2}\right)=$ 1.91 , that is close to 2 .

To check the order of approximation in time, the space step $h$ is fixed $\left(h=10^{-2}\right)$ and time step $k$ is variable. From Table 2 by analogous to (58), formula one gets $\gamma(5$. $\left.10^{-4}, 10^{-3}\right)=0.80$, that is close to 1 . It proves the second order of approximation in space and the first order in time.

5.2. Comparison with Other Approaches. The front fixing method is used in [21] for American put option pricing problem with parameters (56), but with another transformation as follows:

$$
x=\frac{S}{S_{f}(\tau)}, \quad p(x, \tau)=P(S, \tau)=P\left(x S_{f}(t), \tau\right) .
$$

TABLE 2: Convergence in time for fixed space step $h=0.01$ for the problem with parameters (56).

\begin{tabular}{lcccc}
\hline Asset price & True value & 0.0005 & 0.001 & 0.002 \\
\hline 90 & 11.6974 & 11.6984 & 11.6989 & 11.6991 \\
100 & 6.9320 & 6.9319 & 6.9318 & 6.9312 \\
110 & 4.1550 & 4.1555 & 4.1544 & 4.1531 \\
120 & 2.5102 & 2.5103 & 2.5091 & 2.5114 \\
& RMSE & $5.6347-4$ & $9.8234-4$ & $1.4623-3$ \\
& CPU-time (sec) & 0.142 & 0.113 & 0.105 \\
\hline
\end{tabular}

TABLE 3: Comparison with front-fixing method under transformation (59).

\begin{tabular}{ll}
\hline Method & $S_{f}(T)$ \\
\hline Implicit (in [21]) & 0.8615 \\
Explicit (in [21]) & 0.8622 \\
Explicit (proposed) & 0.8628 \\
\hline
\end{tabular}

By the numerical tests, it is shown that the considered scheme is stable for $\mu \leq 24$ while the condition for a stability of the scheme in [21] is $\mu \leq 6$. The results are represented in Table 3. The front fixing method with the logarithmic transformation has a good advantage: weaker condition for the stability. It reduces the computational costs.

Let us compare front fixing method with another approach [31], based on Mellin's transform. The parameters of the problem are $r=0.0488, \sigma=0.3$, and $T=0.5833$.

To compare results of the explicit front fixing method with Mellin's transform [31], we have to multiply our dimensionless value on $E=45$. Then, for Mellin's transform method $S_{f}(T)=32.77$, while for front fixing $\operatorname{method} S_{f}(T)=$ 32.7655 .

Close to maturity, exercise boundary for the American put can be analytically approximated [32] as follows:

$$
S_{f}(\tau) \sim E\left[1-\sqrt{2 \sigma \tau \log \left(\frac{\sigma^{2}}{6 r \sqrt{\pi \tau \sigma^{2} / 2}}\right)}\right] .
$$

This approximation can be used only near expiry date. In Figure 3 , the value of the free boundary for both approaches is presented. Near initial point, front fixing method and analytical approximation (60) give close results.

We compare explicit front fixing method (FF) with $h_{1}=$ 0.001 and $h_{2}=0.002$ and $\mu=5$ for American put with other numerical methods shown in [15] in Table 4 for the following problem parameters:

$$
\begin{gathered}
r=0.05, \quad \sigma=0.2, \quad T=3, \\
E=100, \quad x_{\max }=2 .
\end{gathered}
$$

There are several considered methods as follows:

(i) penalty method (PM) is considered in [21,33];

(ii) Ikonen and Toivanen [34] proposed an operator splitting technique (OS) for solving the linear complementarity problem; 
TABLE 4: Comparison with other methods: put option value for different asset prices.

\begin{tabular}{lccccccc}
\hline Asset price & True value & PM & OS & HW & WK & $\mathrm{FF}\left(h_{1}\right)$ & $\mathrm{FF}\left(h_{2}\right)$ \\
\hline 80 & 20.2797 & 20.2793 & 20.2795 & 20.2803 & 20.2825 & 20.2795 & 20.2793 \\
90 & 13.3075 & 13.3071 & 13.3074 & 13.3075 & 13.3117 & 13.3075 & 13.3074 \\
100 & 8.7106 & 8.7100 & 8.7104 & 8.7103 & 8.7135 & 8.7106 & 8.7104 \\
110 & 5.6825 & 5.6820 & 5.6824 & 5.6823 & 5.6867 & 5.6825 & 5.6823 \\
120 & 3.6964 & 3.6960 & 3.6963 & 3.6965 & 3.7001 & 3.6963 & 3.6963 \\
RMSE & & $4.6690-4$ & $8.9442-6$ & $3.1623-4$ & $3.6116-3$ & $1.0229-4$ & $2.2966-4$ \\
CPU-time & & 25.67 & 11.91 & 4.72 & 4.23 & 25.99 & 4.617 \\
\hline
\end{tabular}

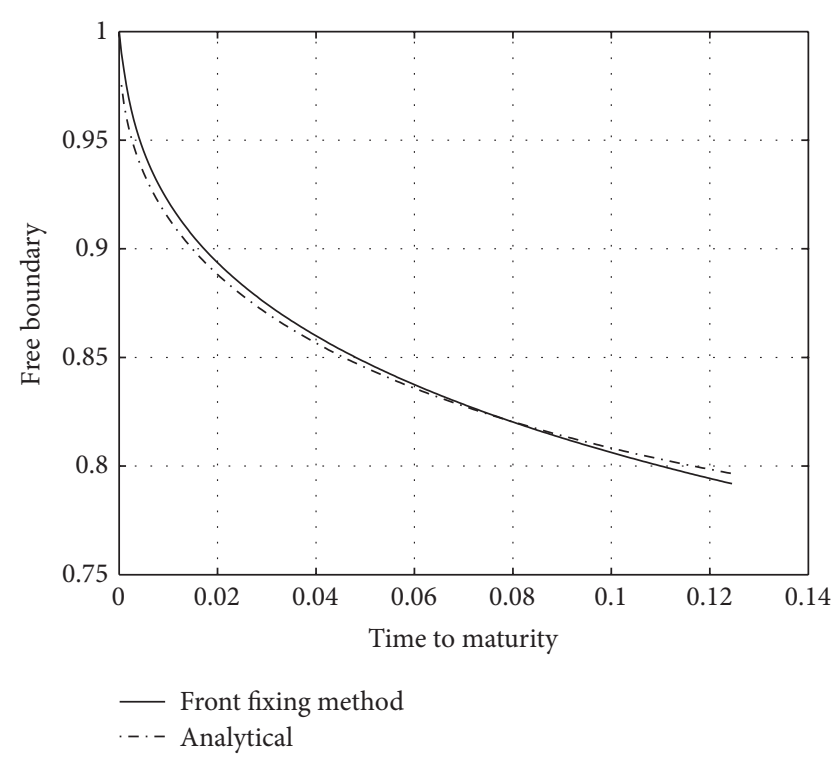

(a)

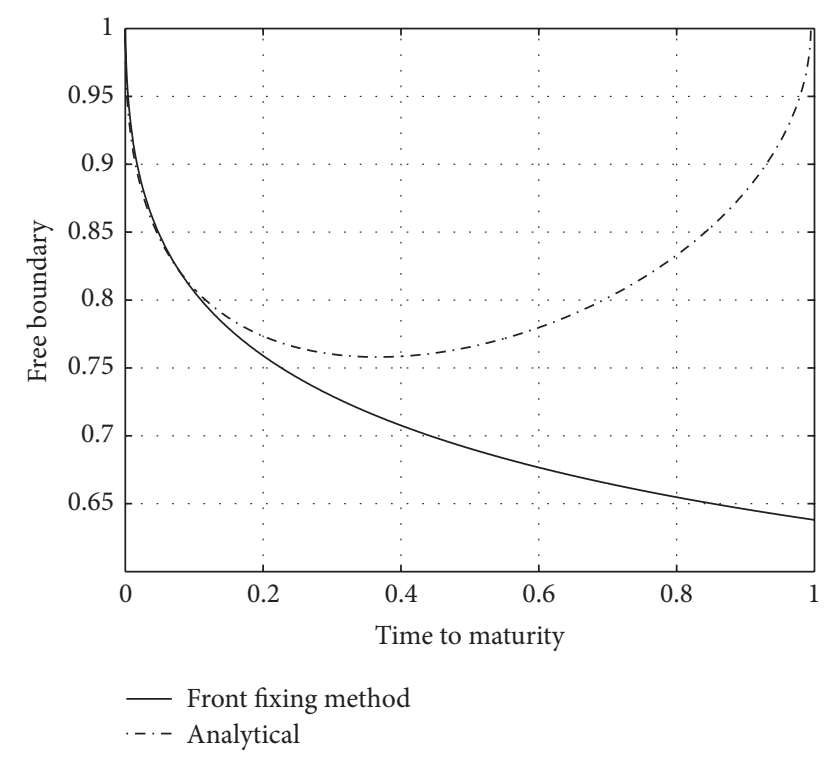

(b)

FIGURE 3: Comparison with the analytical approximation of Kuske close to $\tau=0$ (a) and for $[0, T]$ (b). (iii) Han-Wu algorithm (HW) transforms the BlackScholes equation into a heat equation on an infinite domain [35];

(iv) the front fixing method proposed by Wu and Kwok (WK) [20].

Apart from the previous comparisons showing that the method is competitive, we remark that the proposed scheme here guarantees the positivity of the solution as well as the monotonicity of both solution and the free boundary.

\section{Conclusion}

The front fixing method for American put option is considered. We found that the proposed explicit numerical scheme is stable under appropriate conditions (see Lemma 1) and consistent with the differential equation with the second order in space and first order in time. Moreover, the monotonicity and positivity of the solution and the free boundary under that condition are proved in agreement with Kim's results in [24] about the properties of the theoretical solution. The theoretical study is confirmed by numerical tests. It is shown that the condition (27) is critical; that is, violation of the condition leads to destabilization of the scheme.

By the comparison with other approaches, advantages of the method are discovered. The logarithmic transformation requires weaker stability condition than Landau's transformation, presented in [21]. The explicit calculations with quite weak stability conditions avoid iterations to solve the nonlinear partial differential equation. It makes the method simpler and reduces computational costs.

\section{Conflict of Interests}

The authors declare that there is no conflict of interests regarding the publication of this paper.

\section{Acknowledgment}

This paper has been partially supported by the European Union in the FP7-PEOPLE-2012-ITN Program under 
Grant Agreement no. 304617 (FP7 Marie Curie Action, Project Multi-ITN STRIKE-Novel Methods in Computational Finance).

\section{References}

[1] P. Wilmott, S. Howison, and J. Dewynne, The Mathematics of Financial Derivatives, Cambridge University Press, Cambridge, UK, 1995.

[2] L. Feng, V. Linetsky, J. L. Morales, and J. Nocedal, "On the solution of complementarity problems arising in American options pricing," Optimization Methods \& Software, vol. 26, no. 4-5, pp. 813-825, 2011.

[3] H. P. McKean, "Appendix: a free boundary problem for the heat equation arising from a problem in mathematical economics," Industrial Management Review, vol. 6, pp. 32-39, 1965.

[4] P. van Moerbeke, "On optimal stopping and free boundary problems," Archive for Rational Mechanics and Analysis, vol. 60, no. 2, pp. 101-148, 1976.

[5] R. Geske and H. Johnson, "The American put option valued analytically," Journal of Finance, vol. 39, no. 5, pp. 1511-1524, 1984.

[6] G. Barone-Adesi and R. Whaley, "Efficient analytic approximation of American option values," Journal of Finance, vol. 42, no. 2, pp. 301-320, 1987.

[7] L. W. MacMillan, "An analytical approximation for the American put prices," Advances in Futures and Options Research, vol. 1, pp. 119-139, 1986.

[8] N. Ju, "Pricing an American option by approximating its early exercise boundary as a multipiece exponential function," Review of Financial Studies, vol. 11, no. 3, pp. 627-646, 1998.

[9] M. Brennan and E. Schwartz, "Finite difference methods and jump processes arising in the pricing of contingent claims: a synthesis," Journal of Financial and Quantitative Analysis, vol. 13, no. 3461, 474 pages, 1978.

[10] P. Jaillet, D. Lamberton, and B. Lapeyre, "Variational inequalities and the pricing of American options," Acta Applicandae Mathematicae, vol. 21, no. 3, pp. 263-289, 1990.

[11] J. Hull and A. White, "Valuing derivative securities using the explicit finite difference method," Journal of Financial and Quantitative Analysis, vol. 25, no. 1, pp. 87-100, 1990.

[12] D. J. Duffy, Finite Difference Methods in Financial Engineering: A Partial Differential Equation Approach, John Wiley \& Sons, 2006.

[13] P. A. Forsyth and K. R. Vetzal, "Quadratic convergence for valuing American options using a penalty method," SIAM Journal on Scientific Computing, vol. 23, no. 6, pp. 2095-2122, 2002.

[14] D. Tavella and C. Randall, Pricing Financial Instruments: The Finite Difference Method, John Wiley and Sons, New York, NY, USA, 2000.

[15] D. Y. Tangman, A. Gopaul, and M. Bhuruth, "A fast highorder finite difference algorithm for pricing American options," Journal of Computational and Applied Mathematics, vol. 222, no. 1, pp. 17-29, 2008.

[16] S.-P. Zhu and W.-T. Chen, "A predictor-corrector scheme based on the ADI method for pricing American puts with stochastic volatility," Computers \& Mathematics with Applications, vol. 62, no. 1, pp. 1-26, 2011.
[17] B. J. Kim, Y.-K. Ma, and H. J. Choe, "A simple numerical method for pricing an American put option," Journal of Applied Mathematics, vol. 2013, Article ID 128025, 7 pages, 2013.

[18] H. G. Landau, "Heat conduction in a melting solid," Quarterly of Applied Mathematics, vol. 8, pp. 81-95, 1950.

[19] J. Crank, Free and Moving Boundary Problems, Oxford Science Publications, 1984.

[20] L. Wu and Y.-K. Kwok, "A front-fixing method for the valuation of American option," The Journal of Financial Engineering, vol. 6, no. 2, pp. 83-97, 1997.

[21] B. F. Nielsen, O. Skavhaug, and A. Tvelto, "Penalty and frontfixing methods for the numerical solution of American option problems," Journal of Computational Finance, vol. 5, 2002.

[22] D. Ševčovič, "An iterative algorithm for evaluating approximations to the optimal exercise boundary for a nonlinear BlackScholes equation," Canadian Applied Mathematics Quarterly, vol. 15, no. 1, pp. 77-97, 2007.

[23] J. Zhang and S. P. Zhu, "A Hybrid Finite Difference Method for Valuing American Puts," in Proceedings of the World Congress of Engineering, vol. 2, London, UK, July 2009.

[24] I. J. Kim, "The analytic valuation of American options," The Review of Financial Studies, vol. 3, no. 4, pp. 547-572, 1990.

[25] R. Kangro and R. Nicolaides, "Far field boundary conditions for Black-Scholes equations," SIAM Journal on Numerical Analysis, vol. 38, no. 4, pp. 1357-1368, 2000.

[26] Y.-K. Kwok, Mathematical Models of Financial Derivatives, Springer, Berlin, 2nd edition, 2008.

[27] P. A. Samuelson, Rational Theory of Warrant Pricing, vol. 6, Industrial Management Review, 1979.

[28] R. Company, L. Jódar, and J.-R. Pintos, "A consistent stable numerical scheme for a nonlinear option pricing model in illiquid markets," Mathematics and Computers in Simulation, vol. 82, no. 10, pp. 1972-1985, 2012.

[29] G. D. Smith, Numerical Solution of Partial Differential Equations: Finite Difference Methods, The Clarendon Press, Oxford, UK, 3d edition, 1985.

[30] F. A. A. E. Saib, Y. D. Tangman, N. Thakoor, and M. Bhuruth, "On some finite difference algorithms for pricing American options and their implementation in mathematica," in Proceedings of the 11th International Conference on Computational and Mathematical Methods in Science and Engineering (CMMSE '11), June 2011.

[31] R. Panini and R. P. Srivastav, "Option pricing with Mellin transforms," Mathematical and Computer Modelling, vol. 40, no. 1-2, pp. 43-56, 2004.

[32] R. A. Kuske and J. B. Keller, "Optimal exercise boundary for an American put," Applied Mathematical Finance, vol. 5, pp. 107116, 1998.

[33] J. Toivanen, Finite Difference Methods for Early Exercise Options, Encyclopedia of Quantitative Finance, 2010.

[34] S. Ikonen and J. Toivanen, "Operator splitting methods for American option pricing," Applied Mathematics Letters, vol. 17, no. 7, pp. 809-814, 2004.

[35] H. Han and X. Wu, "A fast numerical method for the BlackScholes equation of American options," SIAM Journal on Numerical Analysis, vol. 41, no. 6, pp. 2081-2095, 2003. 


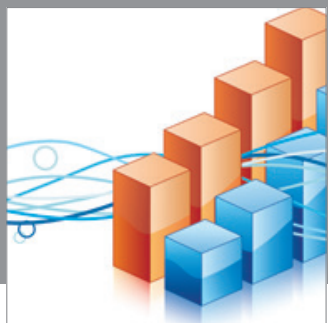

Advances in

Operations Research

mansans

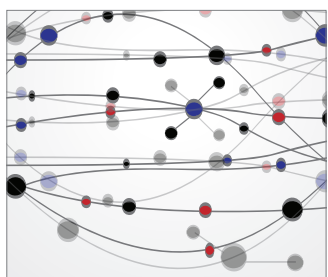

The Scientific World Journal
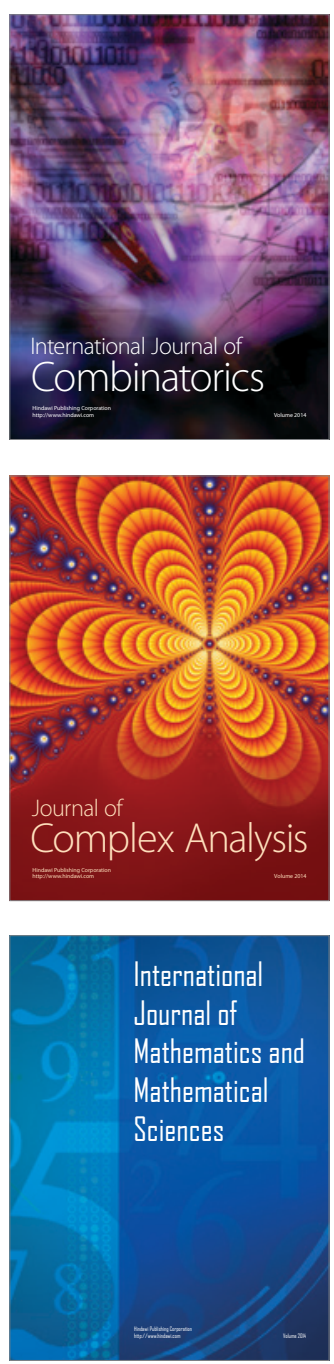
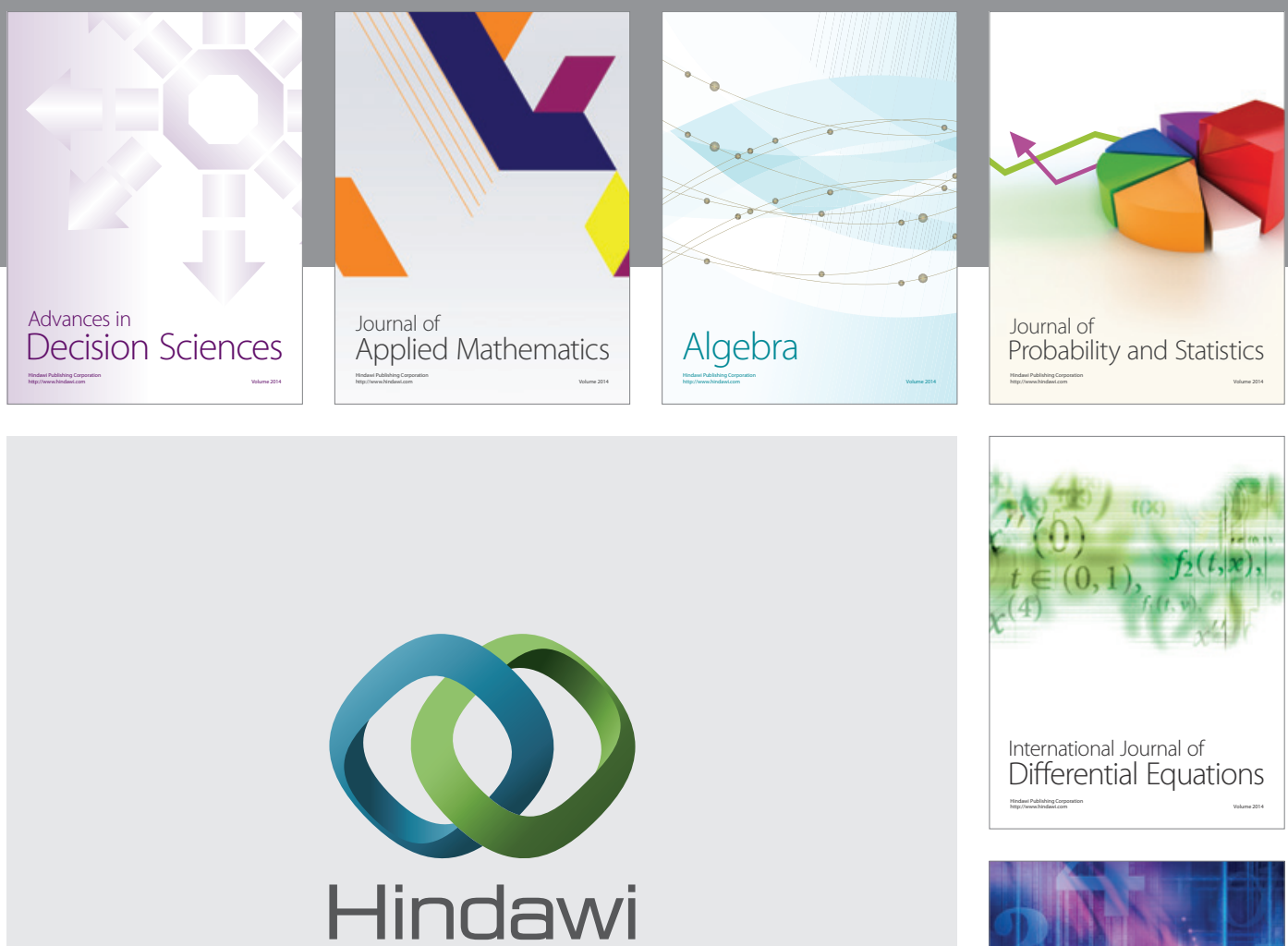

Submit your manuscripts at http://www.hindawi.com
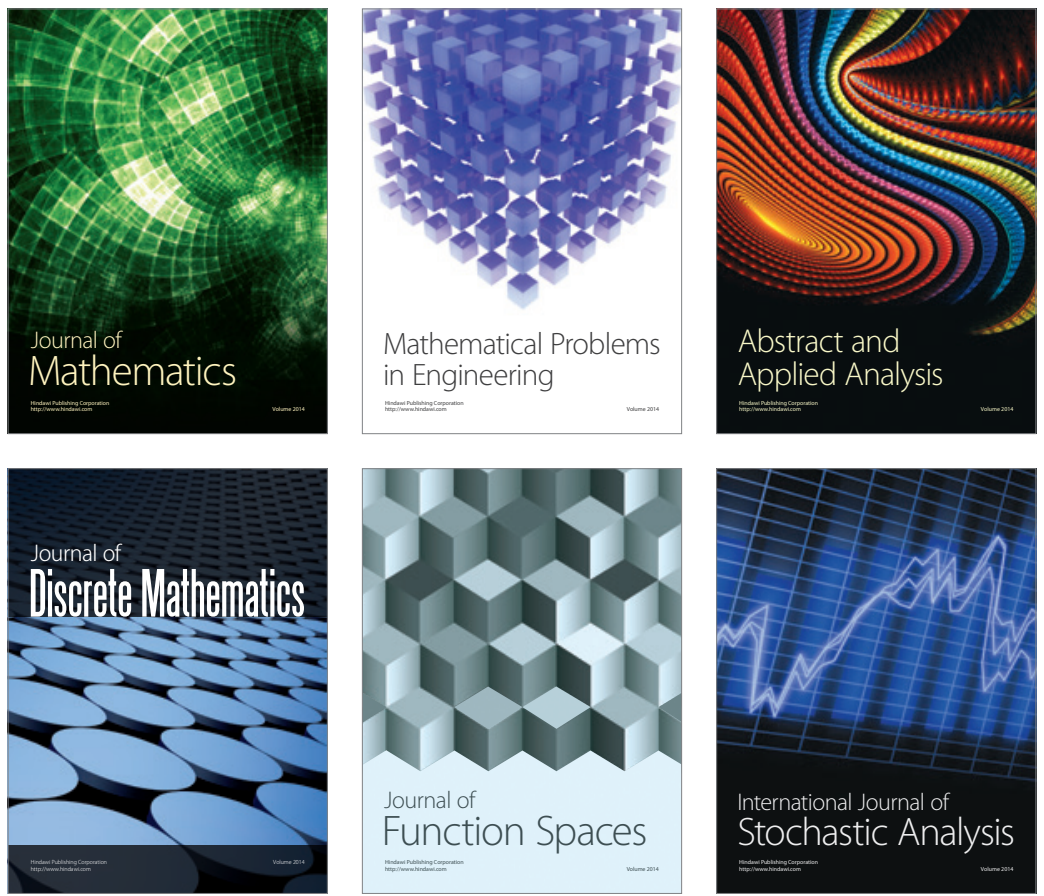

Journal of

Function Spaces

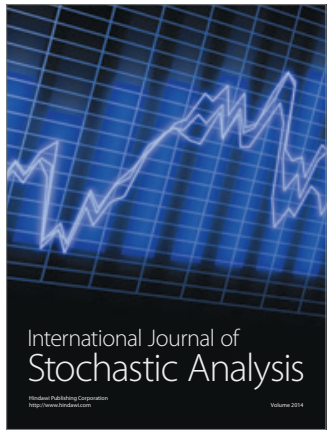

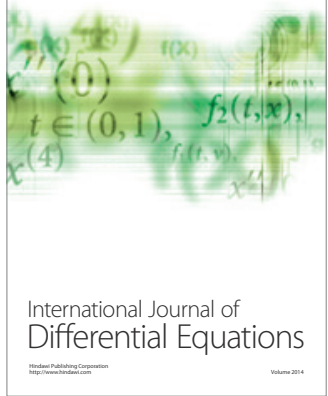
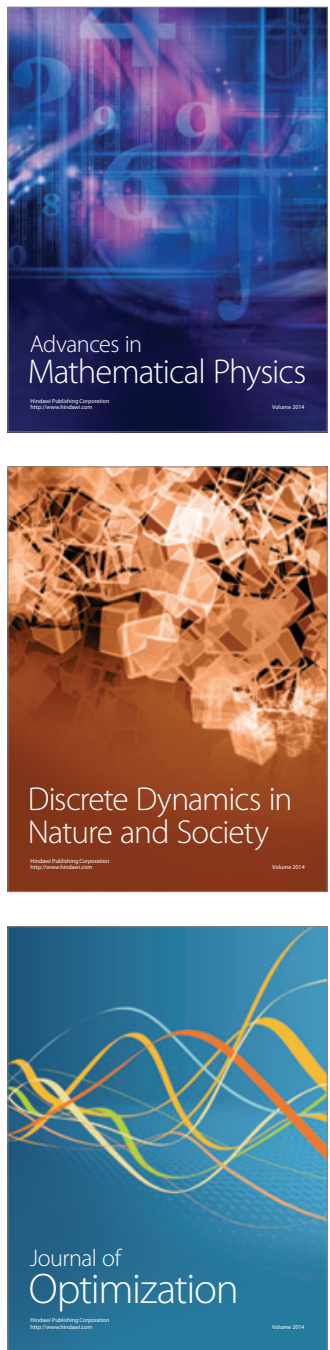